\title{
Effect of Extended Nursing on the Behavioral and Psychological Symptoms and Cognitive Dysfunction of Patients with Moderate and Severe Alzheimer's Disease
}

\author{
MEIHUA CHEN, YU BAI, XIANJU ZHOU, WEI CHEN, DAN HE AND YAN LI* \\ Department of Neurology, the Affiliated Changzhou No. 2 People's Hospital of Nanjing Medical University, 29\# Xinglong Alley, \\ Changzhou, Jiangsu 213000, China
}

Chen et al.: Effect of Extended Nursing on Moderate and Severe Alzheimer's Disease Patients

The purpose of this investigation is to study the effect of extended nursing on the behavioral and psychological symptoms and cognitive dysfunction of patients with moderate and severe Alzheimer's disease. A total of 102 patients with moderate and severe Alzheimer's disease admitted to the People's Hospital of Nanjing Medical University from February 2016 to March 2018 were randomly divided into the observation group and the control group. The control group received routine health education and nursing 
guidance after discharge, while the observation group received additional extended nursing scheme for $1 \mathrm{y}$, including daily living ability training, body function training, language training, memory training, music therapy, psychological nursing support and offline collective activities of patients. The scores of behavioral pathology assessment scale of Alzheimer's disease, Montreal cognitive assessment, and Barthel activities of daily living were compared between the two groups. There was no significant difference in the scores of behavioral pathology assessment scale of Alzheimer's, Montreal cognitive assessment and Barthel activities of daily living between the two groups on admission. One year later, the behavioral pathology assessment scale of Alzheimer's disease scores of the observation group were significantly lower than that of the control group $(\mathbf{p}<\mathbf{0 . 0 5})$, the Montreal cognitive assessment scores and Barthel activities of daily living scores of the observation group were significantly higher than that of the control group $(p<0.05)$. The extended nursing scheme of this study improved the behavioral and psychological symptoms of Alzheimer's disease patients significantly, including anxiety, fear, hallucination, delusion, emotional disorder, aggressive behavior, behavioral disorder and circadian rhythm disorder. It could also improve the cognitive ability of Alzheimer's disease patients significantly, and improve their ability to handle daily life activities.

Key words: Alzheimer's disease, extended nursing, behavioral and psychological symptoms, cognitive function, ability of daily life activities

Alzheimer's disease (AD) was a kind of neurodegenerative disorder prone to the elderly, with pathological changes in the nervous system such as the widened sulcus gyrus, diffuse atrophy of cerebral cortex and neurofibrillary tangles ${ }^{[1]}$. It can affect the health of the patients and social stability seriously. Some neurological dysfunctions such as the irreversible, progressive memory impairment, disability, dementia, aphasia and blindness were found in AD patients ${ }^{[2]}$. Different from the vascular dementia and traumatic dementia, the cause of $\mathrm{AD}$ was unknown, which leads to limited clinical therapeutic measures. With a difficult nursing task and relatively backward domestic nursing experience and concepts, AD nursing remains to be challenging for the nursing doctors, nursing workers, and family members. Active and strategic AD nursing can maximize the function and independence of patients. However, since AD patients were usually nursed at home or nursing home, it was difficult for doctors and nurses to popularize the advanced nursing concepts to $\mathrm{AD}$ patients and their nursing staffs. The extended nursing refers to the continuous follow-up and guidance from the hospital to family or community ${ }^{[3]}$. In this study, an extended nursing scheme was designed and implemented for patients with moderate and severe $\mathrm{AD}$ and their nursing staff. By comparing the behavioral and psychological symptoms and the degree of cognitive dysfunction between the patients receiving the extended nursing and the patients receiving routine nursing, the positive effect and prognostic value of the extended nursing on the AD patients were explored, which could provide a clinical basis and reference for the extension of the extended nursing in $\mathrm{AD}$, and also open up ideas for nursing programs for other neurology diseases. A total of 102 patients with moderate and severe AD admitted to the People's Hospital of Nanjing Medical University from February 2016 to March 2018 were included in this study. Inclusion criteria were, those patients who met all diagnostic criteria related to moderate and severe $\mathrm{AD}^{[4]}$; patients with different degrees of behavioral and psychological symptoms; patients whose families voluntarily participated in the study and signed an informed consent. Exclusion criteria were, those patients who had used or stopped antipsychotic drugs $2 \mathrm{w}$ before treatment; patients with serious diseases of heart, liver, lung, kidney, and other important organs, AD patients combined with other mental diseases. According to the random number method, patients were divided into the control group and observation group, 51 cases each. In the control group, there were 23 males and 28 females (aged 63-82 y, with a mean age of $71.43 \pm 5.14 \mathrm{y}$ ), the course of disease was from 4 to $12 \mathrm{y}$ (mean duration of $7.44 \pm 2.15 \mathrm{y}$ ). In the observation group, there were 24 males and 27 females (aged 62-84 y, with a mean age of $72.38 \pm 4.27 \mathrm{y}$ ); the course of disease was from 5 to $10 \mathrm{y}$ (mean duration of $7.13 \pm 1.64 \mathrm{y}$ ). There was no statistical difference in the baseline data between these two groups. This study was approved by the ethics committee of the hospital.

At discharge, the patients and their families were given routine health education and nursing guidance by the 
nurses according to the condition of the patients. After discharge, they were followed up by telephone one every 3 mo. The follow-up content mainly included self-care ability, mental health and the quality of daily sleep and diet. An extended nursing team was set up, including 1 neurologist and 5 nurses with rich experience in $\mathrm{AD}$ nursing. The team members participated in the study actively and were trained with the extended nursing scheme. In addition to the routine nursing measures of the control group, the following extended nursing measures were taken. Implementation of discharge guidance plan, establishment of Wechat group for the discharged patients, opening of hotline, online provision of $\mathrm{AD}$ rehabilitation, nursing and medication guidance. The Wechat group and hotline mainly have the following functions, inquiry function. After the patients release or call to raise nursing and medication issues within the group, the designated nursing team members should reply within $1 \mathrm{~d}$ and organize appropriate case extension analysis and discussion within the Wechat group. For privacy problems of the individual patients and nursing staff, Wechat private chat or telephone contact was used. When the problems could not be solved remotely, the patients were required to return to the hospital for further consultation. The release of the educational program about $\mathrm{AD}$ nursing knowledge and music and video that can assist patients in the treatment. The design of knowledge education plan was considered comprehensively from the aspects of daily life ability training, body function training, language training, memory training. Many training and teaching videos, which were simple and easy to understand, highly operational, were released once a day in the morning and afternoon, respectively. Moreover, the videos were released circularly to enhance the training effect. The nursing staffs were encouraged to release the training results of the patients to the Wechat group for display, and the nursing team used voice or video to give positive encouragement and guidance. Enhancement of communication among doctors, nurses and patients and if the patients or nursing staff do not appear in group chat for a month, the nursing team members inquired the reasons, informed the significance of active participation in group chat and recommended participation. Psychological nursing work included during the follow-up, nursing team members discussed with patients about their history and expectations, listened to the memories, achievements, life expectation and life experience of the patients and their family ${ }^{[5]}$ to help patients to retrieve their memory and restore hope for the future. In addition, they comforted and dredged the troubles and pains of their family and the nursing staff and to guide the nursing staff to take countermeasures and to assist patients or nursing staff, who have serious psychological symptoms in the follow-up to seek medical treatment for professional psychotherapy. The follow-up personnel improved the support and encouragement of family decision-makers to $\mathrm{AD}$ patients through the introduction of previous cases and psychological counselling ${ }^{[6]}$. Organization of offline activities including group activities such as the sand table games and simple intelligent toys ${ }^{[7]}$ were held every 2 mo to improve patient's yearning for a better life and physical function exercise. The behavioral pathology assessment scale of Alzheimer's disease (BEHAVE-AD) ${ }^{[8]}$ was used in this study. The behavioral and psychological symptoms of the patients were scored by the doctors. BEHAVE-AD has 7 dimensions, anxiety, fear, hallucination, delusion, emotional disorder, aggressive behavior, behavioral disorder, circadian rhythm disorder, a total of 25 items. The score of each item was from 0 to 3 points. The higher the score the more severe the patient's mental behavior symptoms. Montreal Cognitive Assessment Scale (MoCA $)^{[9]}$ and mini mental state examination scale (MMSE) were used to evaluate the cognitive function of the 2 groups. There were 8 dimensions ( 30 scores) in MoCA scale, including naming, language, memory, orientation, attention, executive ability, abstract thinking and delayed memory. The higher the score is, the higher the cognitive ability of the patients. The Barthel activities of daily living (BADL) scale was used for the evaluation of the daily living ability. There were 4 dimensions in BADL scale, including eating, bathing, dressing, and going to the toilet. The score of each dimension was from 1 to 4 points, and the total score was 16 points, the higher the score, the better the basic living activity ability of the patients. SPSS 22.0 software was used to analyze the data, the measurement data were expressed by mean \pm standard deviation, and T-test was used for comparison. $\mathrm{p}<0.05$ means the difference was statistically significant. There was no significant difference in BEHAVE-AD scores between the 2 groups on admission. After 1 y follow-up, the BEHAVE-AD scores of the observation group were significantly lower than that of the control group $(p<0.05)$ as shown in Table 1 . There was no significant difference in MoCA scores between the 2 groups on admission. After $1 \mathrm{y}$ follow-up, the MoCA scores of the observation group were significantly lower than that of the control group $(p<0.05$, Table 2$)$. There was no 
TABLE 1: BEHAVE-AD SCORE OF THE OBSERVATION GROUP AND CONTROL GROUP

\begin{tabular}{lccc}
\hline Group & N & $\begin{array}{c}\text { Admission } \\
\text { score }\end{array}$ & $\begin{array}{c}\text { After 1 year } \\
\text { follow-up }\end{array}$ \\
\hline Observation group & 51 & $19.32 \pm 3.53$ & $7.21 \pm 2.88$ \\
Control group & 51 & $19.47 \pm 3.37$ & $10.37 \pm 2.17$ \\
$\mathrm{t}$ & & 0.219 & 6.258 \\
$\mathrm{p}$ & & 0.827 & 0.000 \\
\hline
\end{tabular}

TABLE 2: MOCA SCORE OF THE OBSERVATION GROUP AND CONTROL GROUP

\begin{tabular}{lccc}
\hline Group & N & $\begin{array}{c}\text { Admission } \\
\text { score }\end{array}$ & $\begin{array}{c}\text { After 1 year } \\
\text { follow-up }\end{array}$ \\
\hline Observation group & 51 & $17.76 \pm 3.68$ & $14.64 \pm 2.38$ \\
Control group & 51 & $17.04 \pm 3.52$ & $12.59 \pm 2.70$ \\
$\mathrm{t}$ & & 1.010 & 4.068 \\
$\mathrm{p}$ & & 0.315 & 0.000 \\
\hline
\end{tabular}

significant difference in BADL scores between the 2 groups on admission. After $1 \mathrm{y}$ follow-up, the BADL scores of the observation group were significantly higher than that of the control group $(\mathrm{p}<0.05)$ as shown in Table 3. AD was a progressive neurodegenerative disease. Due to the gradual loss of the orientation, cognitive function, memory ability, and self-care ability, AD patients suffer from memory deterioration, maze and dementia. Some AD patients may even have the delusion of being killed, violence and other special circumstances and need special care after leaving the hospital. In addition, there were no therapeutic methods that can cure AD totally or improve the symptoms of AD effectively. Drug such as olanzapine, which can improve the memory and control mental symptoms, can only delay the disease ${ }^{[10]}$. Drugs such as memantine and donepezil can also enhance the memory and cognitive function by increasing the levels of neurotransmitter acetylcholine. AD patients have a long course of disease, which can be as long as 15 to $20 \mathrm{y}$. Patients need long-term care for there was a large individual difference in the disease duration ${ }^{[11]}$. Nursing AD patients has caused a heavy burden on society and family. The outline of Healthy China 2030 plan focuses on the long-term care for the elderly with dementia at the end of their lives, improving the quality of life of the elderly with dementia, and safeguarding the dignity of the elderly with dementia. However, due to the fact that the $\mathrm{AD}$ patients were generally cared by their families or communities, the lack of appropriate care means and experience will aggravate the deterioration of $\mathrm{AD}$ patients. How to extend the hospital nursing experience to the families and communities was a subject worthy of clinical consideration. Extended nursing, which concept was born in $1947^{[12]}$, refers to the continuous follow-up and guidance from the hospital to family or community, which guarantees the continuity of the nursing work. The development of the extended nursing in China was relatively slow, and it was still in a small-scale exploratory stage. The outline of China's nursing development plan (2016-2020) issued in 2016 clearly proposed that the extended nursing should be taken as the key development task in the 13 th 5 y plan period. And the medical institutions should be encouraged to gradually improve the content and mode of nursing services, so as to provide multiple extended nursing services for the discharged patients. A systematic and operable extended nursing scheme was designed in this study, whose purpose was to extend the nursing intervention mode in real time and permeability. It mainly relied on the Wechat, family visit and telephone to establish the doctor-patient family communication channel, nurse-patient family interaction mode, and patient-patient mutual aid mode to promote the prognosis of $\mathrm{AD}$, so as to make the $\mathrm{AD}$ clinical nursing spread from hospital to society and allocate the clinical resources and social resources reasonably. The results showed that the behavioral and psychological symptoms of the patients receiving the extended nursing were significantly reduced compared with the control group and the incidence of anxiety, fear, hallucination, emotional disorder, delusion, aggressive behavior, behavioral disorder and circadian rhythm disorder was reduced and slowed. This may be related to the activities of organizing the patients to play sand table games and simple intellectual toys, discussing with the patients about their history and expectations during the follow-up, listening to the patient's and their family memories, achievements, life expectations and life experiences. Moreover, the results of the MoCA scores showed that the cognitive functions of the patients receiving the extended nursing were significantly better than that of the control group after $1 \mathrm{y}$ of the extended nursing. This may be related to the practices of the music and video in Wechat daily push that can assist the patients in the treatment. It is reported that the music therapy can affect the hippocampus, corpus callosum, nucleus accumbens, auditory cortex

TABLE 3: BADL SCORE OF THE OBSERVATION GROUP AND CONTROL GROUP

\begin{tabular}{lccc}
\hline Group & N & Admission score & $\begin{array}{c}\text { After 1 year } \\
\text { follow-up }\end{array}$ \\
\hline Observation group & 51 & $14.53 \pm 1.77$ & $11.41 \pm 1.08$ \\
Control group & 51 & $15.02 \pm 1.82$ & $9.31 \pm 1.52$ \\
$\mathrm{t}$ & & 1.378 & 8.043 \\
$\mathrm{p}$ & & 0.171 & 0.000 \\
\hline
\end{tabular}


and many other brain regions, and $\mathrm{AD}$ was related to the failure and degradation of these brain regions. Therefore, various forms of music therapy can stimulate these brain regions effectively, and slow down their degradation rate, thus delaying the disease ${ }^{[13]}$. Finally, the BADL scale was also used in this study to compare the ability of daily life of the patients, and it was found that the ability of daily life of the patients receiving the extended nursing was significantly better than that of the control group in dressing, eating, going toilet and bathing. The extended nursing scheme carried out the daily life ability training, gave encouragement and guidance to $\mathrm{AD}$ patients, and responded to the questions of the patients and their families positively, all of which were analyzed and popularized as a case. Received high praise among all the patients and their families. There were still some problems to be solved for the extension of extended nursing in $\mathrm{AD}$, such as the improvement of the supporting system and the establishment of the evaluation system. The extended nursing in this study was provided to patients free of charge. In the process of this study, some problems may occur, such as the fatigue and safety of the nursing staffs, the enthusiasm and satisfaction of the nursing staffs, which may interfere with the results of this study. Therefore, the charging and allocation of nursing staffs need to be improved for the extension of extended nursing in $\mathrm{AD}$.

\section{Acknowledgments:}

This work was supported by the National Natural Science Foundation of China (81471338).

\section{Conflict of interests:}

The authors declared no conflict of interest.

\section{REFERENCES}

1. Nowrangi MA. Neuropsychiatric Aspects of Alzheimer Dementia: From Mechanism to Treatment. Psychiatr Clin N Am 2020;43:383-97.

2. Lanctot KL, Amatniek J, Ancoli-Israel S, Arnold SE, Ballard $\mathrm{C}$, Cohen-Mansfield $\mathrm{J}$, et al. Neuropsychiatric signs and symptoms of Alzheimer's disease: New treatment paradigms. Alzheimer's \& Dementia: Translational Research \& Clinical Interventions 2017;3:440-9.
3. Xue Y, Jian S, Tong L, Weixin H. GW25-e4570 Effect of Continuous Nursing Intervention on Quality of Life in Elderly Patients with Chronic diseases. J Am Coll Cardiol 2014;64:C222.

4. Jack Jr CR, Bennett DA, Blennow K, Carrillo MC, Dunn B, Haeberlein SB, et al. NIA AA research framework: toward a biological definition of Alzheimer's disease. Alzheimers Dement 2018;14:535-62.

5. NIA-AA Research Framework: Toward a biological definition of Alzheimer's disease. Alzheimers Dement 2018;14:535-62.

6. Zou G, Li Y, Xu R, Li P. Resilience and positive affect contribute to lower cancer-related fatigue among Chinese patients with gastric cancer. J Clin Nurs 2017;27:e1412-8.

7. Toles M, Song MK, Lin FC, Hanson LC. Perceptions of Family Decision-makers of Nursing Home Residents with Advanced Dementia Regarding the Quality of Communication Around End-of-Life Care. J Am Med Dir Assoc 2018;19:879-83.

8. Di Giulio P, Finetti S, Giunco F, Basso I, Rosa D, Pettenati F et al. The Impact of Nursing Homes Staff Education on Endof-Life Care in Residents with Advanced Dementia: A Quality Improvement Study. J Pain Symptom Manage 2019;57:93-9.

9. Gamba P, Testa G, Staurenghi E, Gargiulo S, Poli G, Leonarduzzi G. The silver lining of cerebrosterol in Alzheimer's disease: the involvement of sirtuin 1 in neuroprotection Free Radic Biol Med 2017;108:S2.

10. Weinstein A, Gujral S, Butters M, Bowie C, Fischer C, Flint A et al. Diagnosing Cognitive Decline: Comparing NIA-AA to DSM-5 Appoaches. Am J Geriatr Psychiatry 2020;28:S79-80.

11. Benek O, Korabecny J, Soukup O. A Perspective on Multitarget Drugs for Alzheimer's disease. Trends Pharmacol Sci 2020;41:434-45.

12. Lopez OL, Kuller LH. Epidemiology of aging and associated cognitive disorders: Prevalence and incidence of Alzheimer's disease and other dementias. Handb Clin Neurol 2019;167:13948.

13. Dualan JJ. Effectiveness of Caregiver-oriented Transitional Care Program in Promoting Preparedness of Caregivers for Home Care of Burn Patients. J Burn Care Res 2020;47:S2-3.

14. Gulliver A, Pike G, Banfield M, Morse AR, Katruss N, Pescud $\mathrm{M}$ et al. Evaluation of the Music Engagement Program for people with Alzheimer's disease and dementia: Study protocol for a pilot trial. Contemp Clin Trials Commun 2019;15:100 419.

This is an open access article distributed under the terms of the Creative Commons Attribution-NonCommercial-ShareAlike 3.0 License, which allows others to remix, tweak, and build upon the work non-commercially, as long as the author is credited and the new creations are licensed under the identical terms

\begin{tabular}{l}
$\begin{array}{l}\text { This article was originally published in a special issue, } \\
\text { "Biomedical Research in Clinical and Preclinical } \\
\text { Pharmaceutics" Indian J Pharm Sci 2020:82(3)Spl issue7;5-9 }\end{array}$ \\
\hline
\end{tabular}

